\title{
Ajuste de resonancia doble en láseres de Brillouin de fibra óptica
}

\author{
V. V. Spirin ${ }^{a}$, C. A. López-Mercado ${ }^{a, *}$, J. L Bueno Escobedo ${ }^{a}$, y A. A. Fotiadi ${ }^{b}, c, d$ \\ ${ }^{a}$ División de Física Aplicada, CICESE, \\ Apartado Postal \#2732, 22860, Ensenada, B. C. México. \\ ${ }^{b}$ University of Mons, Electromagnetism and Telecommunication Department, \\ 31 Boulevard Dolez, B-7000 Mons, Bélgica. \\ ${ }^{c}$ Ioffe Physico-Technical Institute of the RAS, \\ 26 Polytekhnicheskaya Street, St. Petersburg 194021, Rusia. \\ ${ }^{d}$ Ulyanovsk State University, 42 Leo Tolstoy Street, Ulyanovsk, 432970, Rusia.
}

Received 7 November 2020; accepted 17 November 2020

Reportamos una solución técnica simple para un ajuste preciso de la longitud de cavidades cortas de fibra óptica usadas comúnmente en los láseres de Brillouin. La cavidad ajustada del láser es simultáneamente resonante para el bombeo y la emisión de Stokes. La técnica propuesta se basa en registrar la respuesta de Brillouin de la cavidad a un escaneo frecuencial de la emisión del láser de bombeo. Las trazas almacenadas son utilizadas para calcular el exceso de longitud de la cavidad que necesita ser removido de la cavidad original para un ajuste preciso de la resonancia de Brillouin en cualquier longitud de onda preseleccionada del láser de bombeo. Para la comprobación de su funcionamiento se desarrolló el ajuste fino de una cavidad de anillo basada en fibra estándar SMF-28 de Corning de 4 m de longitud. Además, se presenta un análisis detallado del error en el algoritmo de ajuste de la doble resonancia en la cavidad de anillo de fibra del láser de Brillouin de corta longitud. La propuesta presentada es igual de útil para el diseño de láseres de fibra monomodo de espectro óptico ultra-angosto y láseres de fibra de Brillouin Q-switched, así como para aplicaciones que emplean alta potencia en cavidades de fibra que evitan la dispersión de Brillouin estimulada.

Descriptores: áser de fibra de Brillouin; anillo resonador de fibra; resonancia simultánea.

We report a simple technical solution for precise adjustment of short fiber cavities commonly used with Brillouin fiber lasers. The adjusted laser cavity is simultaneously resonant for pump and Stokes radiations. The technique is based on recording the Brillouin response of the cavity to the frequency scanned laser radiation. The recorded traces are used to calculate the excess cavity length that needs to be removed from the original cavity to provide its precise adjustment to the Brillouin resonance at any preselected pump laser wavelength. For a demonstration of the approach, fine adjustment of a $4 \mathrm{~m}$ long ring cavity based on standard Corning SMF-28 fiber is performed. A detailed error analysis of the algorithm for adjustment of double resonance in short-length Brillouin ring fiber laser was presented. The demonstrated approach is equally useful for the design of single mode fiber lasers with ultra-narrow optical spectra, Q-switched Brillouin fiber lasers as well as for applications employing high power fiber cavities free from stimulated Brillouin scattering.

Keywords: Brillouin fiber laser; optical fiber ring resonator; simultaneous resonance.

PACS: 42.81.-i; 42.65.Es; 43.35.Rw.

\section{Introducción}

Este artículo está dedicado a la memoria de E. A. Kuzin, quien realizó una gran contribución reconocida por la comunidad científica mundial al desarrollo de la investigación en el campo de los fenómenos no lineales en las fibras ópticas.

V. V. Spirin, A. A. Fotiadi y C. A. López-Mercado enfatizan la importancia de E. A. Kuzin en la formación y el desarrollo de sus actividades científicas en el campo de la fibra óptica, preservando y desarrollando las enseñanzas recibidas. Evgeny Anatolyevich Kuzin permanecerá para siempre en nuestra memoria como amigo y maestro.

La dispersión estimulada de Brillouin (SBS, por sus siglas en inglés) es el proceso no lineal en las fibras ópticas con el menor umbral, lo que demuestra una extraordinaria propiedad espacio-temporal, como la conjugación de fase óptica en fibras multi-modo, la cual fue descubierta en los trabajos pioneros de E. A. Kuzin en el Instituto Iofee en Rusia [1-4]. La SBS en las fibras ópticas es importante para numerosas aplicaciones como los láseres de fibra, amplificadores de fibra, sensores distribuidos, conjugación de fase, regeneración de señales ópticas, operaciones lógicas completamente ópticas y procesos ópticos de señales de radio frecuencias [1-11].

Recientemente, se han desarrollado láseres de Brillouin de fibra con la llamada cavidad de doble resonancia (DRC), demostrando un bajo umbral, alta pureza espectral y baja intensidad de ruido [12]. Estos láseres son muy prometedores para una variedad de usos especiales, como sensado coherente interferométrico, comunicaciones ópticas, microondas fotónicas y detección radar coherente [13-16].

La emisión de Stokes en estos láseres se genera en una cavidad de anillo de fibra de corta longitud, la cual es simultáneamente resonante para el bombeo y la emisión de Stokes. Esta configuración origina un mínimo umbral de Brillouin. Sin embargo, la operación estable del láser de Brillouin DRC es extremadamente sensible a la desintonización de la resonancia entre la frecuencia del bombeo y el modo de la cavidad. Típicamente, para obtener una operación longitudinal mono-modo (SLM) establecida, se utilizan varios 
tipos de sistemas activos de estabilización, los cuales ajustan la longitud de la cavidad o alteran la frecuencia del láser de bombeo [13-14].

En este trabajo presentamos un procedimiento especial para una precisa configuración de la cavidad de fibra de anillo para lograr la condición de la doble resonancia en cualquier longitud de onda preseleccionada del bombeo. Probamos que la respuesta experimental de Brillouin de la cavidad a un barrido de frecuencia del láser de bombeo permite calcular el exceso de longitud de la cavidad que necesita removerse para desplazar la resonancia de Brillouin a la posición deseada. Demostramos que el algoritmo propuesto proporciona una certera precisión de la localización del pico de resonancia con mediciones ordinarias y con errores de corte.

\section{Resultados experimentales y discusión}

La cavidad del láser de Brillouin debe ser simultáneamente resonante para el láser de bombeo y la emisión de Stokes. El rango espectral libre (FSR) de una cavidad de fibra de algunos metros de longitud puede ser de decenas de $\mathrm{MHz}$. Por lo tanto, el láser de bombeo típico puede conducirse fácilmente a un modo de resonancia de la cavidad mediante ajustes en la temperatura y corriente de inyección. El problema es mantener este encadenamiento a pesar de las desviaciones aleatorias de la longitud de la cavidad y frecuencia del láser. Sin embargo, incluso si se proporciona una coincidencia precisa y estable de la frecuencia del láser de bombeo con uno de los modos resonantes de la cavidad, eso no garantiza la resonancia con la emisión de Stokes desplazada hacia abajo (rojo) para cavidades de corta longitud.

En este apartado presentamos un algoritmo y una técnica experimental para el ajuste de resonancia doble de la cavidad de fibra en cualquier longitud de onda preseleccionada. El esquema experimental se muestra en la Fig. 1.

En el trabajo experimental, la longitud de la cavidad de anillo utilizada fue de $4 \mathrm{~m}$ de longitud, la cual se bombardeó por el láser sintonizable Agilent 81940A con un ancho de línea de $10 \mathrm{kHz}$ y una potencia de hasta $25 \mathrm{~mW}$. El comportamiento del resonador de anillo de fibra óptica es análogo

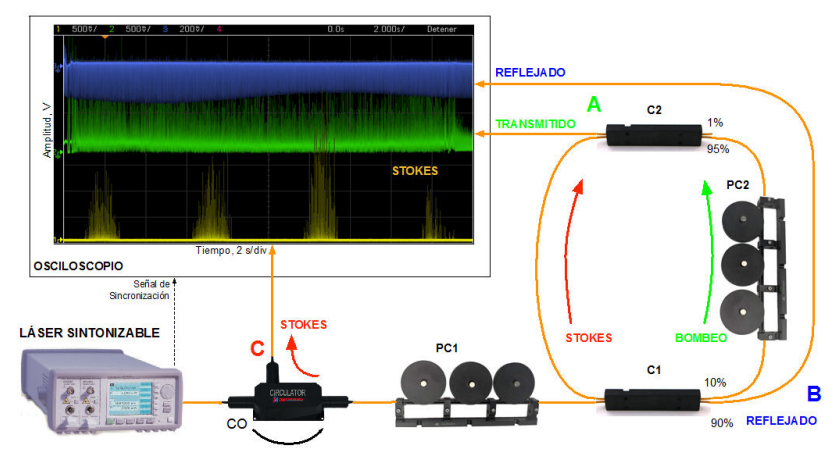

Figure 1. Picos de la doble resonancia generados con un simple barrido en frecuencia del láser de bombeo.

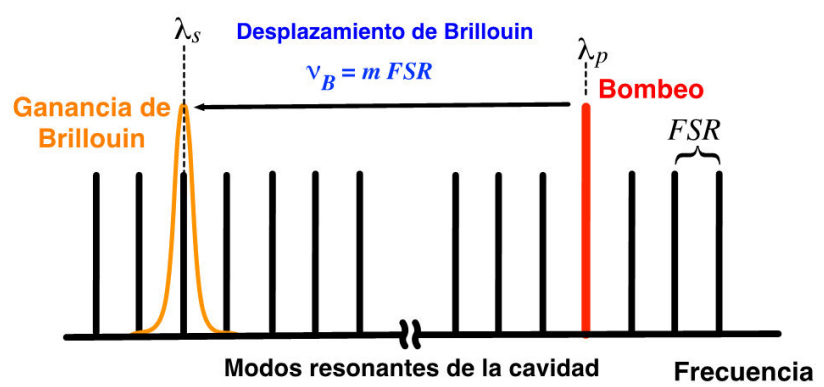

FIGURE 2. Condición de doble resonancia.

a una cavidad lineal Fabry-Perot [17], donde los puertos A y B (ver Fig. 1) son equivalentes a los puertos Transmitido y Reflejado de la cavidad lineal, respectivamente. En la Fig. 1, se presenta gráficamente la resonancia doble en el experimento. La captura realizada en el osciloscopio de Agilent Technologies DSO-X 3024A detalla los momentos precisos de dicha condición. Cuando el láser sintonizable realiza un barrido frecuencial en el intervalo de 1540-1560 nm sus instantes inicial y final se registran con precisión en la traza del osciloscopio para realizar la extrapolación tiempo-frecuencia. Los picos de los Stokes aparecen solo en ciertas longitudes de onda del bombeo, las cuales corresponden a la condición de doble resonancia.

La longitud $L$ de la fibra dentro del anillo define el FSR de la cavidad:

$$
F S R=c / n L
$$

donde $c$ es la velocidad de la luz en el vacío y $n$ es el índice de refracción de la fibra SMF-28 igual a 1.468 en 1550 nm [18].

La doble resonancia se alcanza cuando el desplazamiento de Brillouin $\nu_{B}$ es igual a un múltiplo entero del FSR (ver Fig. 2):

$$
\nu_{B}=m F S R
$$

donde $m$ es un entero, al cual nos referiremos como el orden del pico de Stokes.

Con esta condición, la cavidad se vuelve resonante tanto para el bombeo como para la emisión de Stokes, lo que reduce drásticamente el umbral SBS y conduce a la aparición de picos de Stokes.

Para encontrar los estados de doble resonancia, ajustamos el desplazamiento de Brillouin mediante la sintonización de la longitud de onda del láser de bombeo $\lambda_{p}$, donde el desplazamiento de Brillouin depende de la longitud de onda del bombeo a razón de:

$$
\nu_{B}\left(\lambda_{p}\right)=\frac{2 n V_{A}}{\lambda_{p}}
$$

donde $V_{A} \approx 5800 \mathrm{~m} / \mathrm{s}$ [19] es la velocidad del sonido en la fibra óptica.

Para una medición precisa de la velocidad del sonido, desarrollamos la configuración experimental mostrada en la 


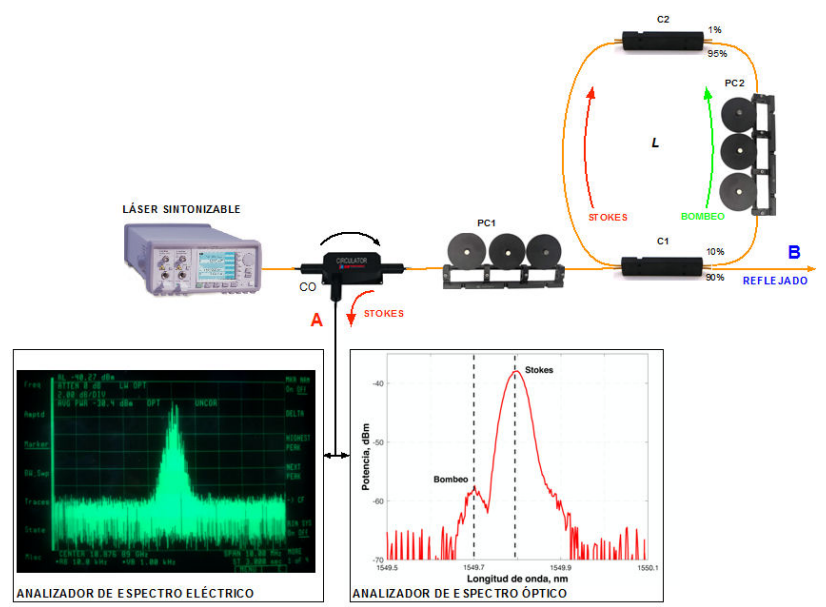

FIGURE 3. Configuración experimental para la medición precisa de la velocidad del sonido en la fibra óptica.

Fig. 3. La emisión del láser sintonizable en una longitud de onda fijada en $1549.7 \mathrm{~nm}$ se inyectó hacia la cavidad de anillo a través del circulador OC y el acoplador C1. Esta longitud de onda fue seleccionada para alcanzar la emisión de Stokes en la cavidad de anillo. La frecuencia de batimiento entre el componente de Stokes y la retrodispersión de Rayleigh del bombeo se registró en el puerto A con un analizador de espectros de RF HP 70908A. La frecuencia de batimiento generada es igual a $10.87689 \mathrm{GHz}$, y empleando la Ec. (3), obtenemos como resultado una velocidad acústica igual a $5740 \mathrm{~m} / \mathrm{s}$, que representa un valor muy cercano al valor teórico esperado.

El acoplamiento de la resonancia entre el barrido del láser de bombeo y el modo de la cavidad ocurre en múltiples intervalos muy cortos. En efecto, el incremento de la potencia del bombeo transmitido en la resonancia es acompañado por un decremento en la potencia reflejada. En la longitud de onda de los Stokes se registraron fuertes pulsos en el puerto C cuando la potencia de bombeo es suficiente para superar el umbral de Brillouin. Estos pulsos se desplazan hacia el rojo por $0.08 \mathrm{~nm}$ de la longitud de onda del bombeo y solo aparecen cuando el desplazamiento de la frecuencia de Brillouin es un múltiplo entero del FSR. Además, existe una perfecta sincronización entre los pulsos de Stokes y los pulsos transmitidos; esta es precisamente la condición de resonancia doble.

Para una estimación precisa de la posición exacta de los picos de doble resonancia, empleamos un procedimiento de promediación utilizando la herramienta computacional MATLAB, así como la conversión temporal a frecuencial, para encontrar la longitud de onda de cada pico generado. En la Fig. 4 se muestra el envolvente de los picos de Stokes después de la promediación de 7 barridos del láser de bombeo, donde cada traza está formada por $1 \mathrm{M}$ muestras, estos son almacenados para la cavidad original no ajustada (traza azul). Debido a que en este algoritmo se utilizó una herramienta elemental de laboratorio como el osciloscopio digital, el procesamiento de los datos es lento, pero eficaz. Dicho tiempo de procesamiento podría reducirse significativamente emplean-

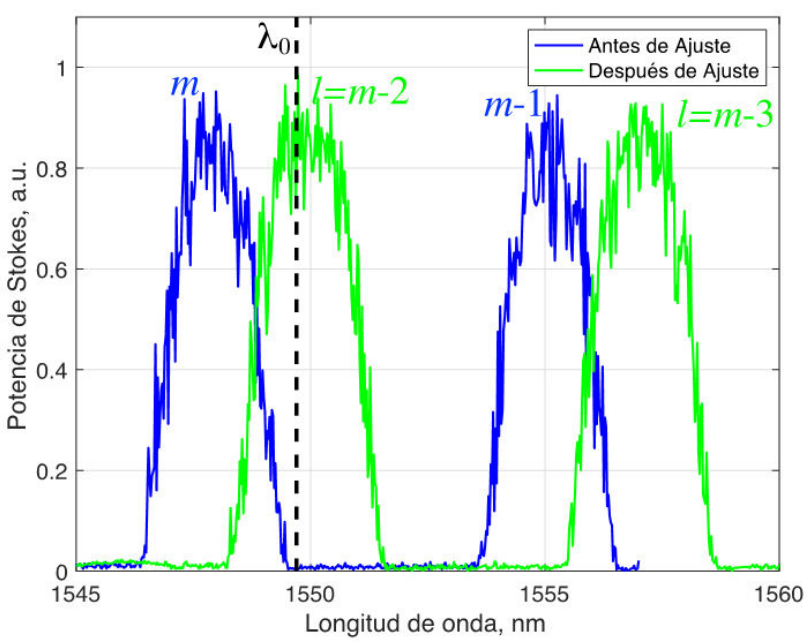

Figure 4. Envolvente de los picos de Stokes después de la promediación de 7 barridos de la cavidad inicial (azul) y después del ajuste (verde).

do equipo capaz de procesar los datos en tiempo real como FPGAs y enfatizando que nuestra técnica es simple y sencilla para ajustar la doble resonancia de las cavidades de fibra óptica.

El orden del pico de la envolvente m puede ser encontrado por medio de las Ecs. (1-3) con la función del entero mas próximo [20]:

$$
m=\operatorname{nint}\left(\frac{\lambda_{m-1}}{\lambda_{m-1}-\lambda_{m}}\right)
$$

donde $\lambda_{m}, \lambda_{m-1}$ son las longitudes de onda centrales de los vecinos cercanos de orden $m$ y $(m-1)$, de los picos de la envolvente dentro del intervalo medido.

Ahora, describimos el procedimiento para el ajuste de la cavidad del láser de Brillouin bombeada con un láser DFB ordinario. El láser DFB de bombeo utilizado emite en una longitud de onda estable de $1549.7 \mathrm{~nm}$. Como se observa en la Fig. 4, la posición de las envolventes en la cavidad inicial se encuentra notablemente fuera de la longitud de onda deseada $\lambda_{0}=1549.7 \mathrm{~nm}$. El pico de las envolventes de Stokes más cercanos a $\lambda_{0}$ tienen una longitud de onda igual a 1547.9 $\mathrm{nm}$ y $1555 \mathrm{~nm}$, que corresponde a $m=219$ y $m-1=218$, respectivamente (ver Fig. 4).

Al emplear las Ecs. (1-3), podemos encontrar que, para desplazar el pico de la envolvente de orden $l$, calculado a partir de la cavidad original y su nueva posición con la longitud de onda central igual a $\lambda_{0}$, debemos de reducir la longitud de la cavidad en $\Delta L$ dada por:

$$
\Delta L=\frac{c}{2 n^{2} V_{A}}\left(m \lambda_{m}-I \lambda_{0}\right),
$$

La Fig. 5 presenta las longitudes $\Delta L$, calculadas para nuestros datos experimentales en función de $m-l$. En teoría, podemos mover hacia la nueva posición cualquier pico de envolvente localizado al extremo derecho de $\lambda_{0}$ [21]. Por ejemplo, podemos mover el pico de orden $218(m-l=1)$ a la posición requerida, pero técnicamente el procedimiento de 


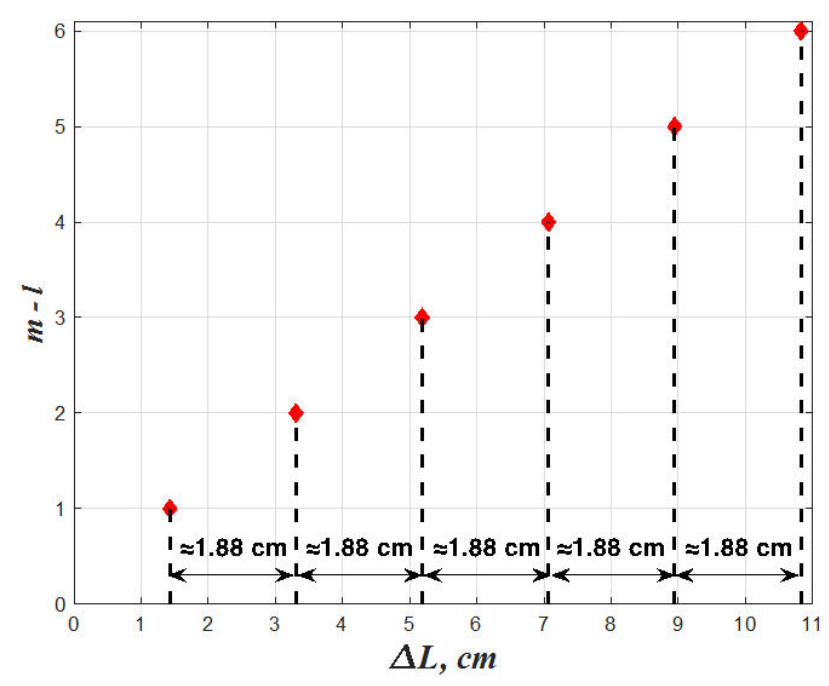

FIGURE 5. Longitud de corte $\Delta L$ vs la diferencia en orden del pico $m-l$.

fusión de la fibra es complicado cuando se requiere un corte menor a $2 \mathrm{~cm}$ de la longitud de la cavidad. En el experimento desplazamos el pico de orden $217(m-l=2)$ hacia la longitud de onda central de $1549.7 \mathrm{~nm}$, mediante un corte en la fibra de $\Delta L=3.3 \mathrm{~cm}$. Como se puede observar en la Fig. 4, el resultado del ajuste de la longitud de la cavidad corresponde con la estimación analítica.

El algoritmo de ajuste de la longitud de la cavidad para lograr la doble resonancia consta de los siguientes pasos:

1) Barrido frecuencial del láser de bombeo a través de la cavidad original, en sincronía con el osciloscopio para almacenar las trazas de Brillouin experimentales.

2) Promediación de las trazas almacenadas y conversión temporal a frecuencial, mediante la herramienta computacional MATLAB.

3) Localización de las longitudes de onda centrales de las envolventes de los picos de Stokes.

4) Cálculo del número de los envolventes de la cavidad original y estimación de la longitud $\Delta L$, la cual debe ser removida de la cavidad para desplazar un pico de resonancia a la longitud de onda preseleccionada.

5) Finalmente, el corte y la fusión de la fibra de la cavidad para reducir su longitud un $\Delta L$.

6) Repetición de los pasos 1-3 para comprobar el desplazamiento del envolvente del pico de Stokes a la longitud de onda preseleccionada.

Si se consideran las incertidumbres del algoritmo para el ajuste de la condición de doble resonancia en el láser de Brillouin de fibra, en cada paso del procedimiento anterior se introducen algunos errores que disminuyen la exactitud de la técnica [21]. La determinación experimental de las longitudes de onda centrales de las envolventes $\lambda_{m-1}$ y $\lambda_{m}$, es acompañada por errores $\delta \lambda_{m-1}$, y $\delta \lambda_{m}$, los cuales dependen de la precisión del equipo usado para para medir la longitud de onda. La incertidumbre estándar del pico de orden $m$ puede estimarse mediante la Ec. (4) con la fórmula de la propagación del error como:

$$
\delta m=\frac{\sqrt{2} \lambda_{m}}{\left(\lambda_{m-1}-\lambda_{m}\right)},
$$

donde $\delta \lambda_{m-1}=\delta \lambda_{m}=\delta \lambda$ son los errores estándar en la determinación de la localización del pico correspondiente.

El error ordinario de la posición del pico $\delta \lambda$ en nuestro experimento es igual a $0.1 \mathrm{~nm}$; sin embargo, al utilizar la promediación adicional sobre varias trazas de la medición, podemos obtener una incertidumbre de $0.05 \mathrm{~nm}$. Incluso, es posible obtener una mayor precisión debido a que el láser sintonizable Agilent 81940A está configurado exactamente para la longitud de onda absoluta típica de \pm 5 pm con una repetitividad de $\pm 1 \mathrm{pm}$

En la Fig. 6 se muestra el error estándar de $m$ en función de la longitud de la cavidad $L$ para diferente precisión en la medición de la posición del pico. Esta dependencia se calcula utilizando las Ecs. (1-3) y (6) para $\lambda_{m}$ de $1550 \mathrm{~nm}$. Este método proporciona un valor absolutamente preciso del orden del pico solo para cavidades muy cortas. Para cavidades con longitudes de 4-8 metros, la incertidumbre de $m$ alcanza valores del 1-2\%. Sin embargo, un error relativamente alto del orden del pico $m$ genera una menor contribución en el error final de la posición del pico de resonancia ajustado.

Además podemos asumir que nosotros realizamos la transferencia del pico de orden $(m-1)$ hacia la posición preseleccionada, la cual se localiza entre los picos de orden $m$ y $(m+1)$. Con esta suposición, la incertidumbre estándar máxima de la reducción de la longitud requerida $\Delta L$ puede estimarse como:

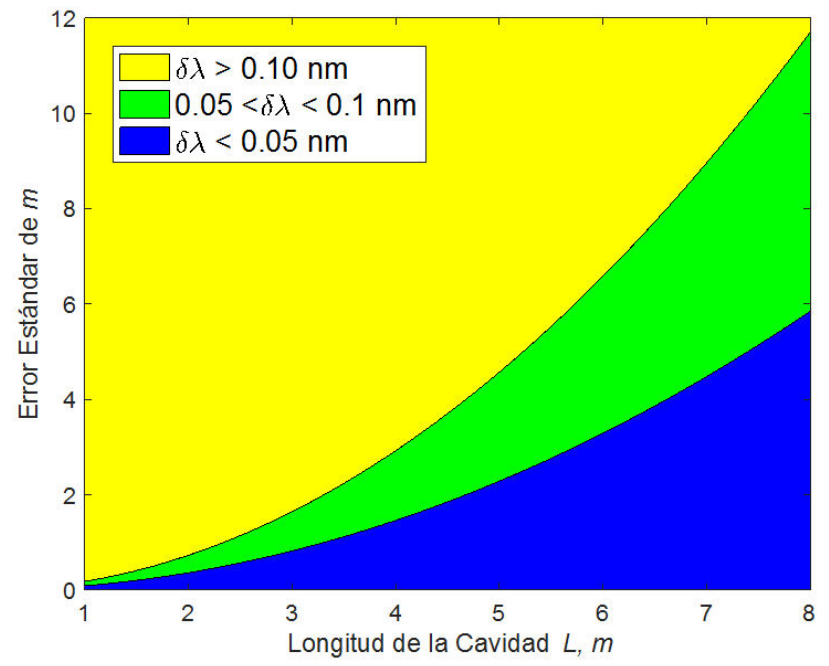

FIGURE 6. Error estándar en el cálculo del orden $m$ del pico de Stokes. 


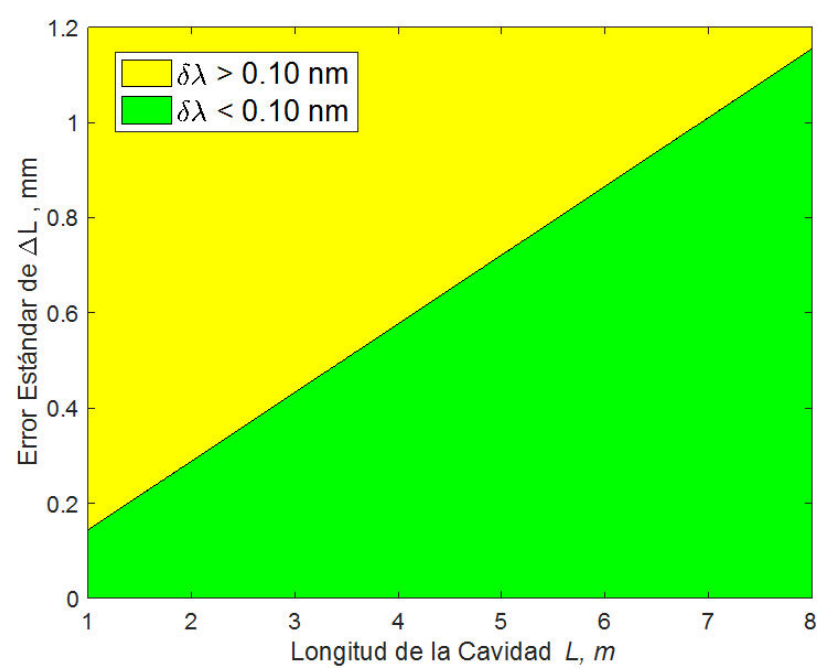

FIGURE 7. Error estándar en las estimaciones de la longitud de corte $\Delta L$ de la cavidad.

$$
\delta \Delta L \cong \frac{c}{2 n^{2} V_{A}}\left(\frac{\sqrt{5} \lambda_{m}}{\lambda_{m-1}-\lambda_{m}}\right)
$$

En la Fig. 7 se presenta la incertidumbre en función de la longitud de la cavidad $L$. Los errores en la estimación de la longitud de corte $\Delta L$ son menores a $0.6 \mathrm{~mm}$ para un error de $0.1 \mathrm{~nm}$ en la posición del pico de resonancia para una cavidad de fibra de $4 \mathrm{~m}$.

Al utilizar la Ec. (5) estimamos la inexactitud de la posición del pico de orden $(m-1)$, el cual hemos desplazado a la longitud de onda $\lambda_{0}$ deseada. Los errores resultantes en la posición del pico debido al corte y los errores de mediciones se presentan en la Fig. 8.

El error en la posición del pico ajustado debido a los errores de medición es igual a 1.6 MHz. Por lo tanto, este error es significativamente menor que el ancho de línea del perfil

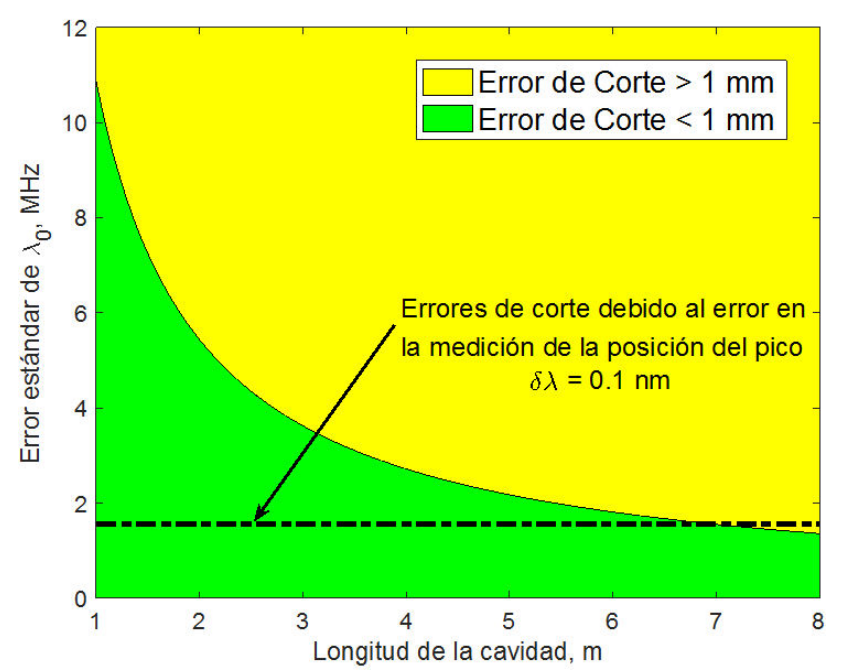

FIGURE 8. Error estándar de la posición del pico desplazado.

de ganancia de Brillouin en la fibra, el cual es de alrededor de $30 \mathrm{MHz}$. Sin embargo, los principales errores del método ocurren debido a las inexactitudes del procedimiento de corte y fusión de la fibra. Afortunadamente, incluso para una cavidad de fibra de $1 \mathrm{~m}$ con un error de corte de $1 \mathrm{~mm}$, se obtiene un error de solo $11 \mathrm{MHz}$ en la posición final del pico desplazado, lo cual resulta bastante razonable para aplicaciones prácticas. Por lo tanto, nuestro algoritmo presenta una certera precisión en la localización del pico ajustado, empleando mediciones ordinarias, así como precisión en el corte y fusión de la fibra.

Además del ajuste de la doble resonancia, la técnica propuesta permite encontrar la longitud de la fibra de la cavidad:

$$
L=\frac{c}{2 n^{2} V_{A}}\left(\frac{\lambda_{m} \lambda_{m-1}}{\lambda_{m-1}-\lambda_{m}}\right) .
$$

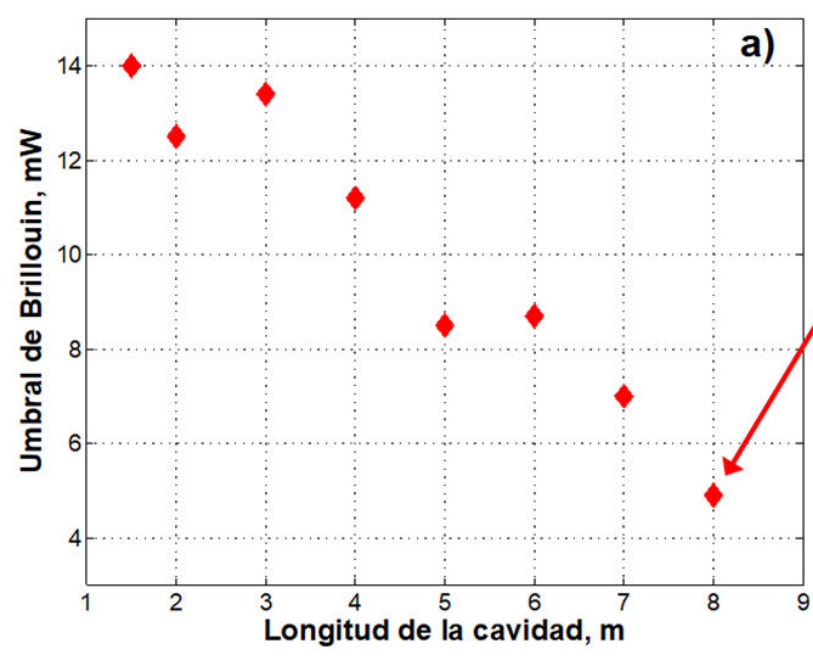

Umbral de Brillouin Umbral en $\lambda$ de en $\lambda$ de Resonancia anti-resonancia

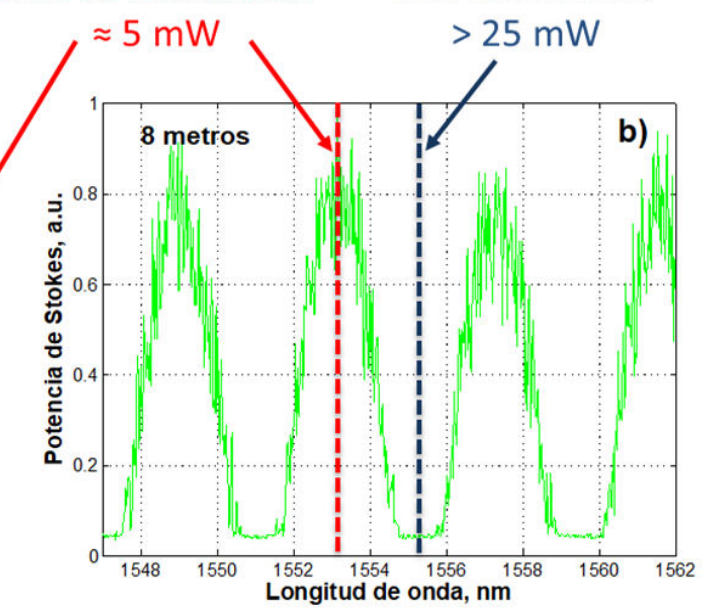

FIgURE 9. Potencia del umbral de Brillouin para diferentes longitudes de cavidades de doble resonancia. 
Sin embargo, el error en la estimación de la longitud de la cavidad con la Ec. (8) es relativamente alto.

El método discutido ha sido probado con cavidades láser de doble resonancia de diferentes longitudes, similares a la presentada en la Fig. 1. El umbral de Brillouin en la condición de resonancia doble se reduce significativamente. La Fig. 9 muestra el umbral de Brillouin en función de la longitud de la cavidad. En la condición de la resonancia simultánea, el umbral de Brillouin llegó a ser igual a 5 y $11 \mathrm{~mW}$ para una cavidad de 8 y $4 \mathrm{~m}$ de longitud, respectivamente.

Por otra parte, para una longitud de onda del bombeo que corresponde exactamente con el mínimo de la dependencia de la resonancia (ver Fig. 2), nunca observamos una emisión de Stokes, incluso para una cavidad de $8 \mathrm{~m}$ de longitud con una potencia máxima del láser de bombeo igual a $25 \mathrm{~mW}$. Así, para estas longitudes de onda de anti-resonancia, el umbral de Brillouin aumenta por lo menos 5 veces.

\section{Conclusiones}

Se presentó un análisis detallado de las incertidumbres de un simple y eficiente algoritmo para el ajuste de la condición de doble resonancia en los láseres de fibra de Brillouin de anillo basados en la medición de la dependencia de resonancia de la emisión de Stokes. Medimos experimentalmente el umbral de Brillouin de la cavidad del láser de fibra adaptada, la cual es simultáneamente resonante para el bombeo y la emisión de Stokes, y alcanza valores de 5 y $11 \mathrm{~mW}$ para cavidades de 8 y $4 \mathrm{~m}$ de longitud, respectivamente. Por otra parte, para longitudes de onda en anti-resonancia, el umbral de Brillouin se incrementa por lo menos 5 veces. Las incertidumbres del procedimiento de ajuste de la resonancia en la longitud de onda preseleccionada comprenden dos partes: los errores debidos a fallas en la medición de la localización de los picos de resonancia y las inexactitudes de la técnica de corte y fusión. Los análisis demuestran que la principal ambigüedad del algoritmo se asocia con las inexactitudes de la técnica de corte. Sin embargo, incluso para el peor de los casos de una cavidad de $1.5 \mathrm{~m}$ de longitud y un error de $1 \mathrm{~mm}$ en el procedimiento de corte y fusión, la técnica propuesta proyecta un error de solo $7 \mathrm{MHz}$ en la posición del pico de resonancia ajustado, menor que el ancho de línea del perfil de ganancia de Brillouin en la fibra, el cual mide alrededor de $30 \mathrm{MHz}$. Una mayor exactitud en el procedimiento de corte puede proporcionar una localización precisa del pico. En este caso, el error de la localización del pico final es determinada por los errores experimentales en la localización del pico inicial. Con un error experimental ordinario de $0.1 \mathrm{~nm}$, el error en la ubicación del pico final es tan solo de $1.5 \mathrm{MHz}$ para cualquier longitud de la cavidad. Por lo tanto, el algoritmo proporciona una ubicación exacta del pico ajustado con mediciones ordinarias y errores de corte. Los datos almacenados también permiten estimar la longitud de la fibra de la cavidad con una exactitud de 2-4\%.

Demostramos que la condición de doble resonancia de los láseres de Brillouin DRC de corta longitud depende de la longitud de la cavidad. Además, experimentalmente logramos desplazar una envolvente del pico de resonancia hacia una longitud de onda $\lambda_{0}=1549.7 \mathrm{~nm}$, con una simple reducción de un $\Delta L=3.3 \mathrm{~cm}$.

Como resultado, la técnica para el ajuste de la doble resonancia en el láser de Brillouin de fibra es simple y robusta ante los errores de la medición y el procedimiento de corte, y puede ser muy útil para el diseño de láseres de Brillouin de cavidad de doble resonancia, así como para aplicaciones que requieren resonadores de fibra de alta potencia libres de SBS. Hemos presentado dos enfoques para el diseño de láseres de Brillouin de fibra doblemente resonante de modo longitudinal único estabilizado pasivamente.

\section{Agradecimientos}

A CONACYT, por patrocinar a J.L. Bueno Escobedo como posdoctorado.
1. E. A. Kuzin, M. P. Petrov, y A. A. Fotiadi, Phase conjugation by SMBS in Optical Fibers.- in Optical Phase Conjugation. Ed. M. Gower, D. Proach. (Springer Verlag, 1994), pp. 74-96.

2. E. A. Kuzin y M. P. Petrov, Stimulated Brillouin scattering in optical fibers and wavefront reversal. Fizika Tverdogo Tela (English translation in Solid State Physics, USA), 25 (1982) 334.

3. E.A. Kuzin y M.P. Petrov, Prospects for controlling laser beams by means of stimulated Brillouin scattering. Pis'ma $\mathrm{v}$ Zh.Tekh.Fiz. (English translation in Sov.Tech.Phys.Lett. USA), 11(7), (1985), pp. 389-393.

4. E. A. Kuzin, M. P. Petrov y B. E. Davydenko, Phase conjugation in an optical fibre. Opt Quant Electron 17 (1985) 393. https://doi.org/10.1007/BF00619565

5. D. A. Grukh, A. S. Kurkov, I. M. Razdobreev, y A. A. Fotiadi, Self-Q-switched ytterbium-doped cladding-pumped fibre laser.
Quantum Electronics. 32 (2002) 1017. https : //doi.org/ $10.1070 /$ QE2002v032n11ABEH002339

6. A. A. Fotiadi, Random lasers: An incoherent fibre laser. Nature Photonics. 4 (2010) 204. https://doi.org/10.1038/ nphoton.2010.76

7. V. V. Spirin, J. Kellerman, P. L. Swart, y A. A. Fotiadi, Intensity noise in SBS with injection locking generation of Stokes seed signal. Opt. Express. 14 (2006) 8328. https : //doi.org/ $10.1364 / \mathrm{OE} .14 .008328$

8. E. A. Kuzin, J. Sancher-Mondragon, V. A. Vyslouch, J. A. Andrade-Lucio y R. Rojas-Laguna, Regeneration of optical signals by fiber logic elements operating through stimulated Raman scattering, Optical Fiber Communications, OFC., San Jose, CA, USA, 1996, pp. 153-154. https://doi.org/10. $1109 /$ OFC.1996.907722 
9. E. A. Kuzin, and M.P. Petrov, Optical logic elements using optical fiber, Avtometriia, (1986) pp. 87-92.

10. E. A. Kuzin, M. P. Petrov, y V. V. Spirin. A logic inverter using stimulated Raman scattering of light in an optical fiber, Pisma v Zhurnal Tekhnischeskoi Fiziki, 12 (1986) 406.

11. A. A. Fotiadi, R. Kiyan, O. Deparis, P. Megret, y M. Blondel, Statistical properties of stimulated Brillouin scattering in single-mode optical fibers above threshold. Opt. Letters. 27 (2002) 83. https://doi.org/10.1364/OL. 27.000083

12. L. F. Stokes, M. Chodorow, y H. J. Shaw, All-single-mode fiber resonator Opt. Letters. 7 (1982) 288. https://doi.org/ $10.1364 /$ OL .7 .000288

13. S. Norcia, S. Tonda-Goldstein, D. Dolfi, J. P. Huignard, y R. Frey, Efficient single mode Brillouin fiber laser for low-noise optical carrier reduction of microwave signals. Opt. Letters. 28 (2003) 1888. https://doi.org/10.1364/OL.28. 001888

14. J. Geng, S. Staines, Z. Wang, J. Zong, M. Blake, y S. Jiang, Highly Stable Low-Noise Brillouin Fiber Laser With Ultranarrow Spectral Linewidth. IEEE Photon. Technol. Letters. 18 (2006) 1813.https://doi.org/10.1109/LPT.2006. 881145

15. S. Molin, G. Baili, M. Alouini, D. Dolfi, y J. P. Huignard, Experimental investigation of relative intensity noise in Brillouin fiber ring lasers for microwave photonics applications. Optics Letters. 33 (2008) 1681. https://doi.org/10.1364/ OL.33.001681

16. J. L. Bueno Escobedo et al., Self-injection locking of the DFB laser through an external ring fiber cavity: Application for phase sensitive OTDR acoustic sensor, Results in Physics, 7 (2017) 641. https://doi.org/10.1016/j.rinp. 2017.01 .013

17. C. A. López-Mercado et al., Locking of the DFB laser through fiber optic resonator on different coupling regimes, $O p$ tics Communications 359 (2016) 195. https://doi.org/ 10.1016/j.optcom.2015.09.076

18. Disponible en: http://www.corning.com/ opticalfiber/index.as

19. L. Thevenaz. Brillouin distributed time-domain sensing in optical fibers: state of the art and perspectives. Frontiers of Optoelectronics in China. 3 (2010) 13. https://doi.org/10. 1007/s12200-009-0086-9

20. V. V. Spirin et al., Single cut technique for adjustment of doubly resonant Brillouin laser cavities, Optics Letters. 38 (2013) 2528. https://doi.org/10.1364/OL.38.002528

21. C.A. López-Mercado et al., Accuracy of single-cut adjustment technique for double resonant Brillouin fiber lasers. Optical Fiber Technology. 20 (2014) 194. https: //doi.org/10. $1016 / j \cdot y o f t e .2014 .01 .011$ 